\title{
VALIDATION PROCESS OF THE ISIS CFD SOFTWARE FOR FIRE SIMULATION
}

\author{
C. Lapuerta, F. Babik, S. Suard, L. Rigollet \\ Institut de Radioprotection et de Sûreté Nucléaire (IRSN) \\ DPAM, SEMIC, LIMSI BP3 - 13115 Saint Paul-lez-Durance, France \\ \{celine.lapuerta, fabrice.babik, sylvain.suard, laurence.rigollet\}@irsn.fr
}

\begin{abstract}
Fire propagation constitutes a major safety concern in nuclear facilities. In this context, IRSN develops a CFD code, named ISIS, dedicated to the fire simulations. This software is based on a coherent set of models that can be used to describe a fire in large, mechanically ventilated compartments. The system of balance equations obtained by combining these models is discretized in time using fractional step methods, including a pressure correction technique for solving hydrodynamic equations. Discretization in space combines two techniques, each proven in the relevant context: mixed finite elements for hydrodynamic equations and finite volumes for transport equations. ISIS is currently in an advanced stage of verification and validation. The results obtained for a full-scale fire test performed at IRSN are presented.
\end{abstract}

\section{INTRODUCTION}

Among the potential incidents in a nuclear facility, fire represents a considerable hazard. If left unmitigated, a fire can damage critical safety elements, and may even lead to radioactive release in the facility or in the environment if containment is compromised. Radioactive material is contained by subdividing the facility into partially or totally sealed compartments, and interconnecting them through a ventilation system of passive components (filters) and active components (fans). The active components maintain under-pressure in zones where any accidental radioactive release must be captured and filtered by the ventilation system. In association with facility sectorization, the containment configuration also contributes to safety, preventing damage caused by fire and smoke from spreading to adjacent rooms.

Fire modeling has improved continuously since its beginnings in the 1950s. It initially centered on correlations with experimental data or observations, then on analytical work, before focusing on numerical simulation, with the first generation of codes producing "zone-based" simulations. The next generation was based on applying scientific computing to fluid mechanics, which made it possible to describe a fire by numerically solving the conservation equations governing flow. Known as CFD (Computational Fluid Dynamics), this approach is now the main focus of simulation research and is widely used in the engineering and safety fields. The computer code ISIS, described below, is based on this approach.

Modeling nuclear facility fires is particularly difficult as the natural complexity of fire development is compounded by coupled interaction with flows in the ventilation system. The originality of the ISIS code is its capacity to take into account this coupling. The thermodynamic pressure is related with the mass flow rate for ventilation vents by the mass balances.

The first section of this article presents the physical models used in ISIS to describe a fire, and the numerical solution of the resulting balance equation system. The second part examines the method used to verify and validate the code. The third section describes in detail the ISIS validation on an integral fire conducted in the DIVA facility at IRSN. 


\section{CFD CODE ISIS}

The code ISIS is in open source, available at https://gforge.irsn.fr/gf/project/isis. The code copes with a wide range of applications including laminar or turbulent flows, possibly reactive, governed by incompressible, low Mach number Navier-Stokes equations. The set of physical models available in ISIS are detailed in (Physical Modelling in ISIS, 2010) . We present here the physical modelling used for the industrial application described before. A brief description of numerical methods are given.

\subsection{Physical modelling}

During a fire in an enclosure, natural convection gives rise to flows in the enclosure atmosphere: gases heat up around the flame, rise in plume, transfer heat to adjacent structures and, once cooled in the plume are roughly a few meters per second, well below the velocity of sound. In other words, the characteristic time scale of this convective motion is small compared to the transit time for a sound wave in the system: the flow in question is said to have a low Mach number. Consequently, it can be shown (Majda, 1985) that the flow obeys a specific system of equations, derived from the equations of motion for compressible flows and differing only by the equation of state. The pressure characterizing the fluid (i.e. a term in the equation of state), referred to as the thermodynamic pressure, is assumed uniform within the space of the enclosure (but potentially variable in time). As in the case for incompressible flows, the momentum equation still has a pressure gradient term, allowing the velocity field to satisfy the mass balance. This gradient is estimated by using the dynamic pressure, variable in space and time. When the flow is isothermal and homogenous, this model is identical to the NavierStokes equations for incompressible flows.

Fire modeling is characterized by the wide range of spatial scales that must be considered. In a containment of several hundred cubic meters, there are fluctuations in flow that modify the flow characteristics at the observed scale, known as turbulent fluctuations. Given current computing capacity, it is not feasible to simulate the smallest of these scales, which must instead be modeled. For ISIS, this is achieved using methods known as "single-point statistics", which have been widely used by engineers for a few decades. In the first step, averaging the balance equations (Navier-Stokes, energy balance, mass balance for chemical species, etc.) leads to a system that displays average fields, but leaves certain terms dependent on their fluctuations. These terms are then expressed according to the average field, by relationships known as "closure relations". In the momentum balance equation, this operation displays an apparent viscosity (or turbulent viscosity), which must then be calculated using a turbulence model. For the current version of ISIS, the standard model $k-\varepsilon$ is used, completed by a term that represents the effects of buoyancy. This model is described in the literature as a firstorder model with two balance equations, which complement the air flow equation system, i.e. the mass and momentum balances (Poinsot, 2001). Near the wall, where viscous effects are dominant, the twoequation models for a high Reynolds number are not valid. Standard wall functions are used to take into account the boundary layers (Cox, 1995).

Turbulent combustion is modeled in a simplified manner by a single reaction, involving three chemical species: fuel, oxygen, and products in the presence of a neutral gas. This reaction is considered complete and irreversible. Assuming an instantaneous reaction between the fuel and the oxygen, the mean chemical reaction rate is controlled solely by the turbulent mixture. This model is typically referred to as the EBU (Eddy Break-Up) model (Cox, 1995). The resulting system is generally governed by three mass balance equations (for the fuel, oxygen, and products). While the three chemical species in the mixture are assumed to have the same mass diffusion, it can be shown by linear combination that only two of the equations are independent; in ISIS, these equations involve the fuel mass fraction and a variable (called the mixture fraction), combining concentrations in such a way that the chemical reaction term is eliminated.

The general form of the energy equation for a multicomponent reacting system is extremely complex and is usually simplified. For low velocity flows, such as fires, many widely-used simplifications are possible. The viscous dissipation, the mixture kinetic energy and the work due to buoyancy forces can 
be ignored in comparison to the heat flux vector. Moreover, for our applications, we suppose that for all chemical species the diffusion coefficient is the same and that the thermal and species diffusivities are equal (Poinsot, 2001). As for turbulence modelling, near the wall, the convective flux is computed from wall functions using the laminar and turbulent Prandtl number (Cox, 1995). Finally, the conduction mechanisms in walls are modelled by a Fourier's equation.

During a fire, due to the large geometrical dimensions of the physical area involved, radiation contributes significantly to heat transfer and therefore to flame development. The finite volume method (Chai, 1994), (Raithby, 1990) is selected for the integral application. This method is nowadays a popular method extensively used in computational fluid dynamics for different and complex situation. Neglecting the scattering effect and using the gray gas assumption, the radiative transfer equation is reduced in a transport equation following the directions of propagation. This approach is valid for semi-transparent and optically thick media. The absorption coefficient of the medium depends on gas temperature and composition, according to experimentally established correlations (Smith, 1982), as well as the soot volume fraction.

The originality of the ISIS code is its capacity to take into account the effect of ventilation on the pressure. The thermodynamic pressure and the mass flow rate for ventilation vents are related by the mass balances in the compartment and in the ventilation branch where an aeraulic resistance is taken into account. This point is major for simulating fire in nuclear plants. Indeed, during a fire in a confined and mechanically ventilated compartment, the variations of thermodynamic pressure inside the fire room modify mass flows at the admission and at the extraction branches, involving potential containment problems.

\subsection{Numerical properties}

The equation system solved by ISIS comprises eight tightly coupled balance equations: the mass balances (global, fuel, and mixture fraction), the momentum and energy balances, the transport equations for turbulent energy $k$ and its dissipation rate $\varepsilon$, and finally, the radiation intensity balance equation describing radiative heat transfer. Mathematically, these equations can be divided into two distinct groups: hydrodynamic relationships (momentum and mass balances or Navier-Stokes equations) on one hand, and the remaining balance equations on the other.

The second group of equations is discretized in time and in space using a finite volume method to obtain schemes that achieve a good compromise between cost and accuracy, and ensures that unknowns stay within their physical boundaries (positive temperature and concentrations between 0 and 1); second-order upwinding techniques are used to accurately take into account fast spatial variations in unknowns (e.g. at the flame front or close to the walls), without stability loss.

The hydrodynamic problem is incompressible in nature, due to the low Mach number assumption, by which density is independent of pressure. It is discretized in space using a finite element technique that satisfies the compatibility properties between velocity and pressure necessary for stability. Unlike finite volume schemes with staggered meshes, this technique also makes it easy to use meshes that are locally unstructured due to the geometry involved or refinement. To ensure coherence with the finite volume discretizations, the approximation selected is low-order and conforming. An approximation of the momentum equation convective terms was developed in order to conserve kinetic energy (Ansanay, 2010), which is necessary to describe very low viscosity flows with large meshes.

To make ISIS more efficient and robust, temporal discretization is carried out with a fractional step scheme (Babik, 2005), i.e. the equations are solved in sequence. This type of scheme has many advantages: cost per time step is reduced, discrete systems are easier to solve, and adding new balance equations to the system is simple. On the other hand, the ISIS scheme is not as accurate as an implicit method, and this must be offset by reducing the time step. 
Finally, ISIS is based on the scientific computing development platform PELICANS. Developed at IRSN, and now available as open-source software (https://gforge.irsn.fr/gf/project/pelicans), PELICANS offers a library of software components, consisting of "building blocks" for implementing numerical methods. ISIS is entirely parallelized via this platform, for both the assembly and solution of discrete systems. In addition, it is possible to resolve linear systems using certain algorithm libraries, available as open-source software, including PETSc (Argonne National Laboratory), thereby ensuring that computer code performance meets current standards.

\section{VERIFICATION \& VALIDATION APPROACH}

In view of this central role played by computer predictions, a major question rises, namely the assessment of the reliability of the simulations. More precisely, the problem posed is how to assess the degree of accuracy and validity of results given by a computer code; this is the aim of the verification and validation process $(\mathrm{V} \& \mathrm{~V})$. Nowadays, the most commonly referenced and agreed literature on this topic is probably the work of (Roache, 1998), of (Oberkampf, 2002) and the (AIAA guide, 1998). Among the concepts clarification brought by these authors, the most important may be the following basic definition for verification and validation:

- Verification: the process of determining that a model implementation accurately represents the developer's conceptual model and the solution of the model.

- Validation: the process of determining the degree to which a model is an accurate representation of the real world from the perspective of intended uses of the model.

The relationships between development, verification and validation are illustrated in Figure 1.

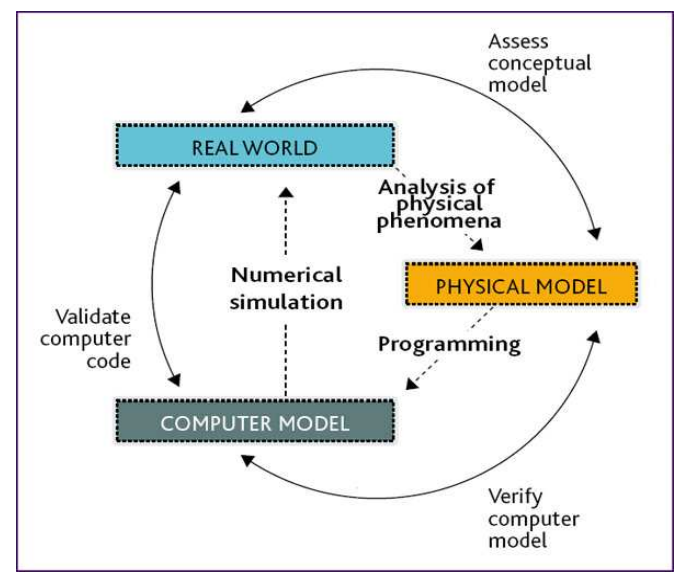

Figure 1: Sequences involved in development, verification and validation of a simulation code

(AIAA guide, 1998)

These both steps are used for each new development in ISIS code.

\subsection{Verification}

The verification tests in ISIS enable to evaluate the consistance properties and the convergence of numerical schemes used. The principal methods are the following:

- Analytic solution verification: the solution of a system is analytically known. The initial and boundary conditions are given by the reference solution. The major limitation of this technique 
is that governing equations admitting an analytical solution are usually obtained for physical problems much simpler than the real situation of interest, often leaving the physical phenomena uncoupled.

- Manufactured solution verification: this method consists in building an analytical source term by applying the considered partial derivation operators to a given solution. The manufactured solution is then the exact solution of the partial differential equations plus the analytical source terms. This approach enables to verify each implemented partial differential equation and the coupling between several equations.

- Numerical benchmark verification: benchmark solutions are solution to physical problems which may be considered as accurate and reliable, because obtained and published by a wide panel of researchers using different and often high-performance numerical methods. However, these verification tests, in most cases and up to now, are in two dimensions and essentially address laminar and incompressible flows.

Examples of verification tests for ISIS code are given in (Suard, 2006).

\subsection{Validation}

Code validation basically consists of comparing the numerical results with experimental data representing the real world situation of interest. ISIS uses a validation methodology defined and recommended by (AIAA, 1998; Oberkampf, 2002; Roache,1998). The first step consists in breaking up the complex engineering system of interest in several sub-system of less complexity:

- Complete system: it is the final application for which the code must be validated. The phenomena are strongly coupled: turbulence, combustion with soot, radiation transfer, ... The spatial domain can be varied between 100 and $5000 \mathrm{~m}^{3}$ and the fire time between 500 and 5000 s.

- Sub-system: it is a subset of the complete application. The number of phenomena is reduced as well as the spatial domain and the studied time in order to decrease time computation and to verify the convergences.

- Model problem: this step of the validation consists in tests where the geometry is often bidimensional, and the problem is steady. The coupling between phenomena are simplified: turbulence/combustion, turbulence/buoyancy, ...

- Unit problem: it is an academic test. The physical process must be known and experiments must be precise and well-documented. Only one phenomena is validated by unit test.

The validation of the ISIS code is built to simulate a fire in mechanically ventilated enclosures. An example of validation phases is illustrated in Figure 2.

In the following, we will focus on the integral case, the others test-case being detailed in (Validation of ISIS, 2010). 


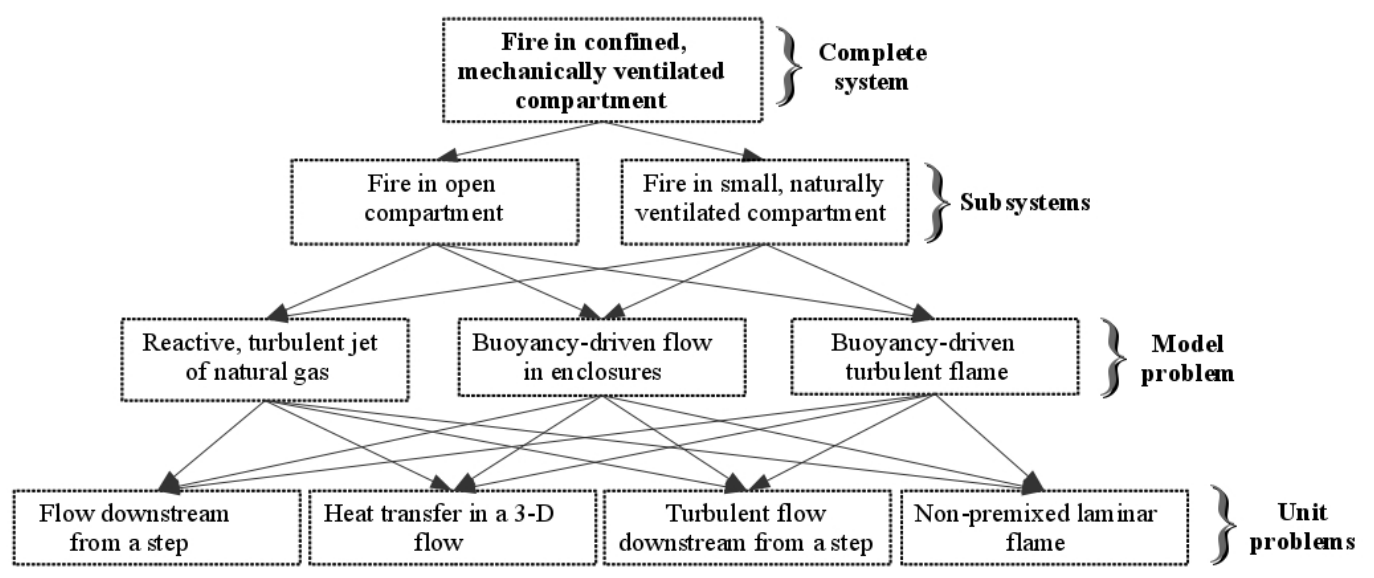

Figure 2: Various validation phases of the ISIS code

\section{SIMULATION OF INTEGRAL CASE}

Several integral tests conducted at the IRSN Fire Test Laboratory and consisting of a large scale fire scenarios in confined and mechanically ventilated compartments have allowed to progress in the validation process of the ISIS code. This section presents some of the numerical results obtained by simulating one of these scenarios named Prisme Source D1 (Pretrel, 2004).

This fire test was the subject of a benchmark exercise proposed in the framework of the PRISME ${ }^{1}$ OECD analytical working group (Audouin, 2010). One of the objectives of the PRISME experimental program was to analyse the effect of the ventilation flow rate on the heat released rate in a confined and mechanically ventilated compartment.

In the following, the numerical results obtained by ISIS for the PRISME Source D1 test are presented.

\subsection{Test description}

This fire experiment was in a single compartment of dimension $5 \times 6 \times 4 \mathrm{~m}^{3}$. The walls were $30 \mathrm{~cm}$ thick and made of concrete and the ceiling was insulated by rock wool panels of $5 \mathrm{~cm}$ thickness. The ventilation system included intake and exhaust branches which were located in the upper part of the compartment (see Figure 3). The initial ventilation flow rate was fixed at $560 \mathrm{~m}^{3} / \mathrm{h}$. The pool fire of $0.4 \mathrm{~m}^{2}$ area was a circular pan filled with a liquid fuel called TPH.

The burning rate used for the simulation is given by the experiment (see Figure 4). At the beginning, the burning rate rapidly increased like in open pool fire situations. Then, as the oxygen concentration decreased the mass loss rate decreased too, which implied a long steady state. The fire stopped by lack of fuel.

1 The acronym PRISME comes from the French phrase Propagation d'un incendie pour des scénarios multilocaux élémentaires, which in English can be translated as "Fire propagation in elementary multi-room scenarios" 


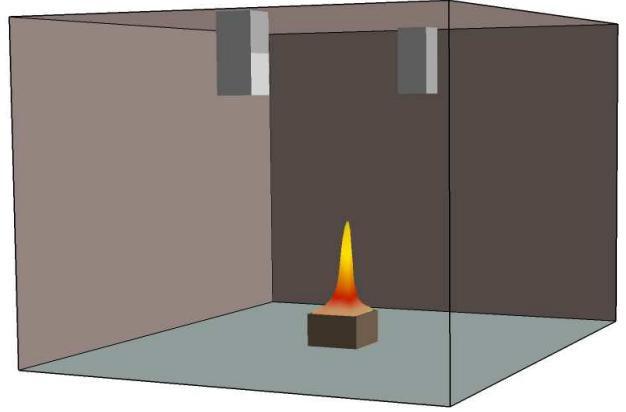

Figure 3: Configuration of the computational domain

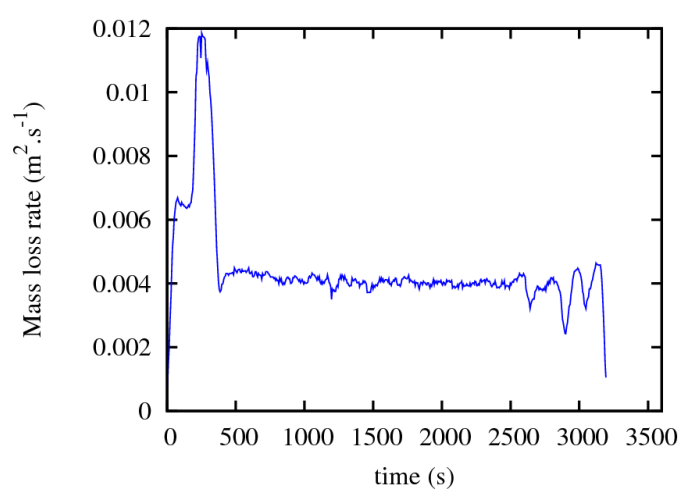

Figure 4: Experiment burning rate

\subsection{Numerical parameters}

The simulated time is 4000 s. To reduce computational time, the time step is adaptive according to numerical criteria. This strategy enables to have a large time step during the steady phase observed in Figure 4.

In order to simulate correctly the flow near walls, the mesh is thinner in this zone. The flame zone required too a small mesh to compute correctly the combustion and the radiative transfer. The simulation is performed with 236000 cells.

\subsection{Results}

Numerical results are compared with the experimental measurements. Table 1 gives an indication of the experimental uncertainties. The understanding of complex phenomena occurring during this test is limited and therefore cannot permit to determine the various experimental uncertainties with a great precision. In the following, the experimental uncertainties are reported on the figures.

\begin{tabular}{|c|c|c|c|c|c|c|}
\hline Quantity & $\begin{array}{c}\text { Gas } \\
\text { temperature }\end{array}$ & $\begin{array}{c}\text { Oxygen con- } \\
\text {-centration }\end{array}$ & $\begin{array}{c}\text { Wall } \\
\text { temperature }\end{array}$ & pressure & $\begin{array}{c}\text { Admission } \\
\text { flow rate }\end{array}$ & $\begin{array}{c}\text { Exhaust flow } \\
\text { rate }\end{array}$ \\
\hline $\begin{array}{c}\text { Relative } \\
\text { uncertainty }\end{array}$ & $10 \%$ & $2 \%$ & $15 \%$ & $30 \%$ & $10 \%$ & $10 \%$ \\
\hline
\end{tabular}

Table 1: Summary of the relative experimental uncertainties

Different points are studied to validate the ISIS code on the integral case: ventilation mass flows, gas and wall temperature, oxygen concentration. These quantities are major for the safety of an installation.

\subsubsection{Ventilation}

It can be seen from experimental measurements that the time variation of the compartment pressure is divided into three distinct phases:

i) an unsteady state phase, during which the pyrolysis rate increases then falls to its nominal value; the observed pressure variations and pressure peak are thus caused by the heat release rate in the compartment and the aeraulic resistance of the mechanical ventilation system. 
ii) a quasi-steady state combustion phase, where the pyrolysis rate is constant. An equilibrium is established between the energy produced by combustion and the heat lost to the walls, on one hand, and heat extracted by the ventilation system on the other. The pressure is nearly constant during this phase;

iii) a final extinguishing phase, during which the flow of evaporated fuel decreases considerably, creating an imbalance between the energy supplied by the fire and the energy lost as heat. The pressure initially falls, but rises once again to equilibrium level in the second half of this phase, due to air supplied by the ventilation system.

On Figure 5 we can see that ISIS was able to correctly compute these variations.

The computed mass flow rates in vents are in accord with the experiment (see Figure 6). After ignition, an inversion of flow at the intake branch occurs. The inversion is caused by a rapid increase of the pressure due to fire ignition during this unsteady-state phase. Numerically, the both inversions are correctly computed. On Figure 6, the code slightly over predicts the flow in the exhaust branch. This variation can be explained by the fact that in the experiment the flow is measured inside the duct whereas in ISIS the flow is directly computed in the outlet.

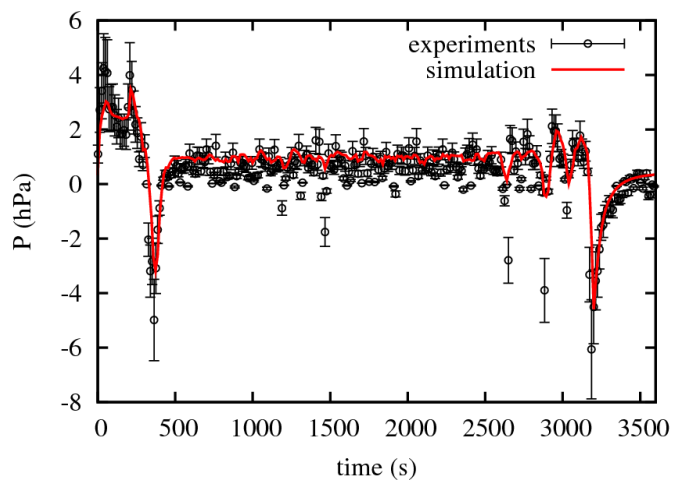

Figure 5: Evolution in time of the thermodynamic pressure in the room

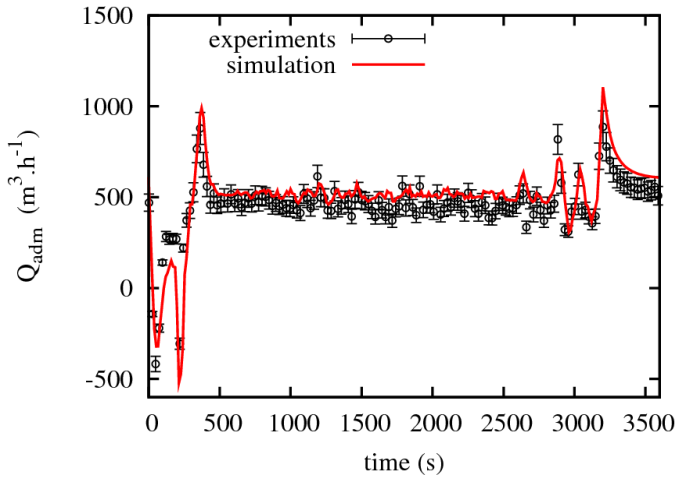

(i) admission

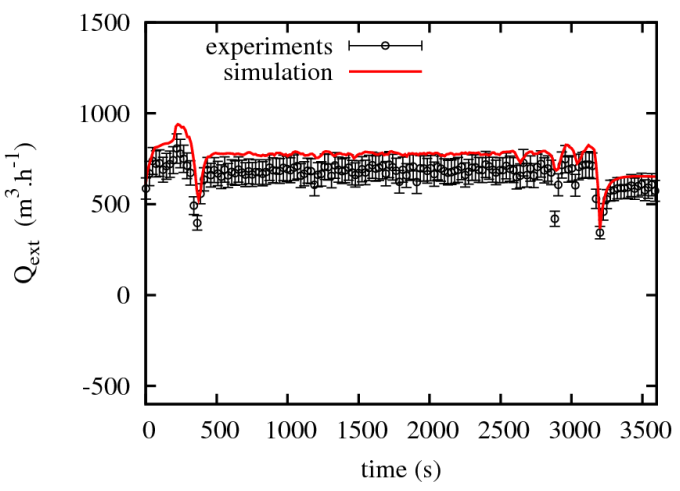

(ii) exhaust

Figure 6: Evolution in time of the admission and exhaust flow rate 


\subsubsection{Gas and wall temperature}

The time evolution of the gas temperature in the room far from the flame is shown in Figure 7. At fire beginning $(\mathrm{t}=40 \mathrm{~s})$, gases heat quickly in the plume. A hot gas layer composed of combustion products and smoke occurs by natural convection close to the ceiling. During the steady phase, this hot zone expands almost completely occupying the room and impact of the vents are depicted by Figure 7 (iii). At fire end, the ventilation cools the gases.

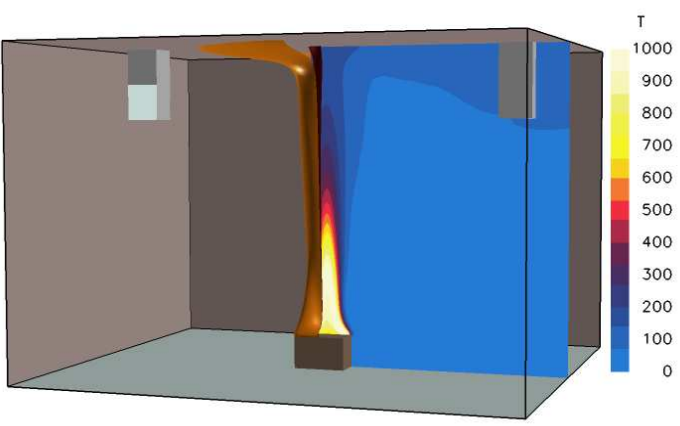

(i) $\mathrm{t}=40 \mathrm{~s}$

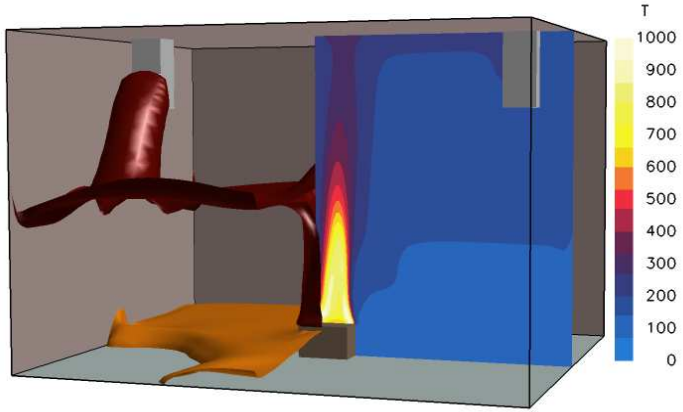

(iii) $\mathrm{t}=2000 \mathrm{~s}$

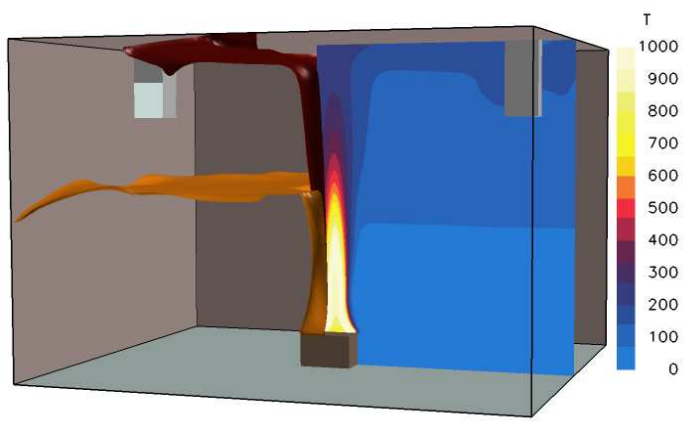

(ii) $\mathrm{t}=80 \mathrm{~s}$

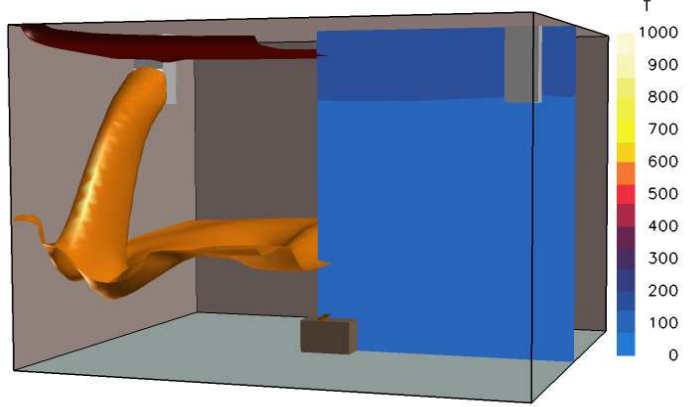

(iv) $\mathrm{t}=3225 \mathrm{~s}$

Figure 7: Temperature evolution in the room: isotherms $100^{\circ} \mathrm{C}$ and $150^{\circ} \mathrm{C}$ at left and temperature field (in ${ }^{\circ} \mathrm{C}$ ) at $\mathrm{x}=0.3 \mathrm{~m}$ at right.

Figure 8 represents the time evolution of the temperature far from the flame for several heights. The temperature is slightly under-predicted by the code during the fire but remains in the range given by the experiment uncertainties. At the fire beginning, the temperature peak is correctly computed.

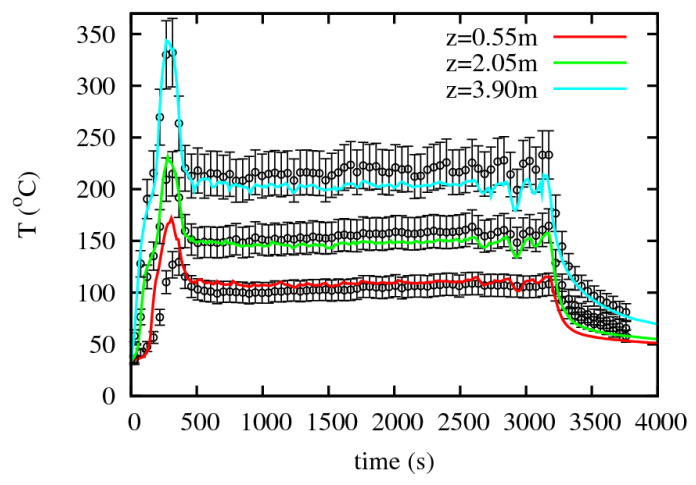

Figure 8: Time-dependent change in gas temperature at various heights in compartment 
The ISIS code enables a computation of the wall temperature from the flux equality at the wall interface. Figure 9 shows the time evolution of the wall temperature for several heights. The numerical results are in accord with the experiments.

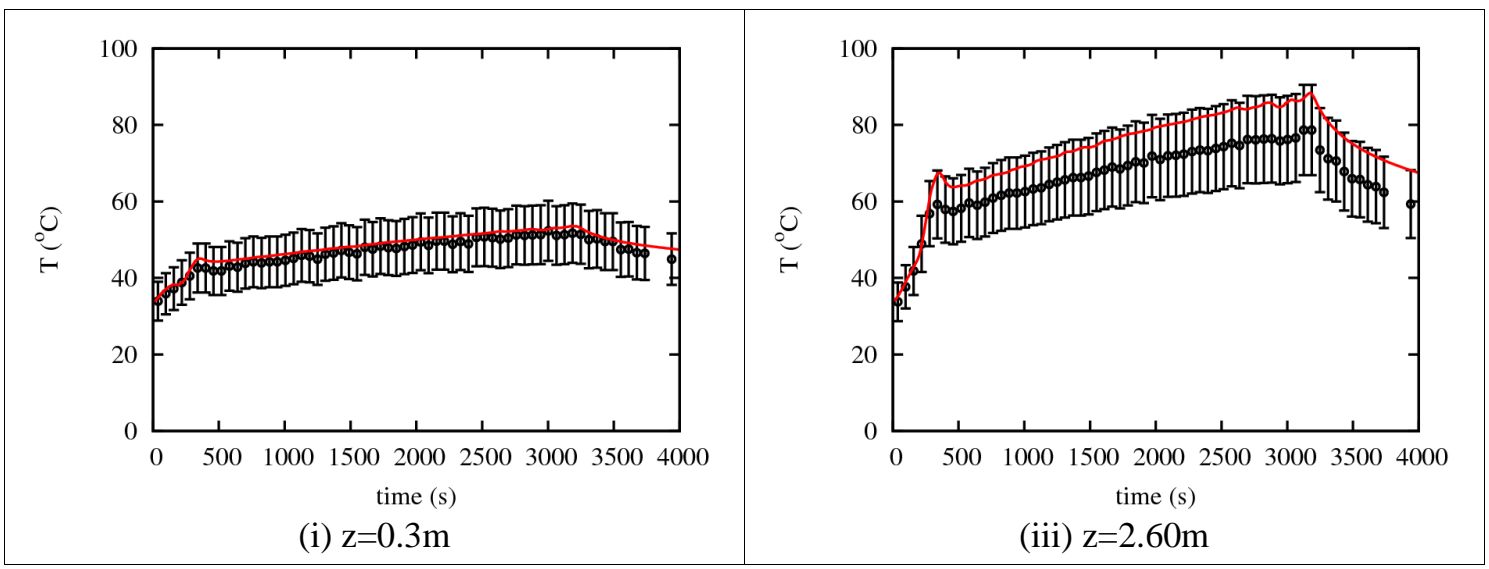

Figure 9: Time-dependent change in wall temperature in two points (lower and upper part)

\subsubsection{Oxygen concentrations}

For oxygen concentration, the numerical results are in accord with the experiments (see Figure 10). The oxygen concentration plays an important role on the fuel mass loss rate and consequently on the fire duration. As the oxygen concentration decreases, the burning rate decreases too and the fire time is longer as a fire scenario in a well ventilated room.

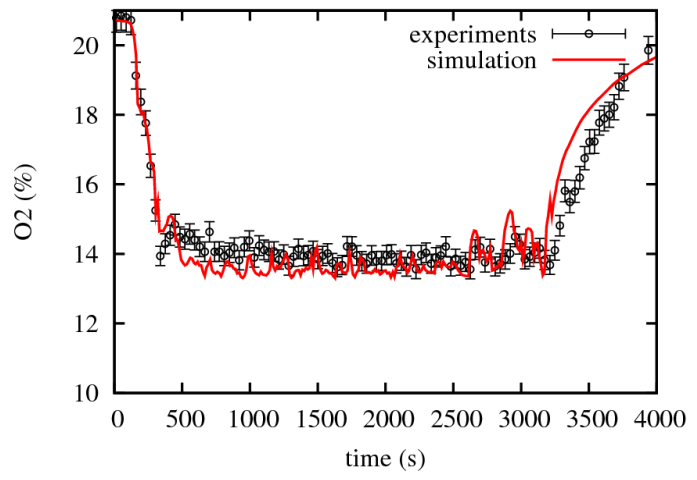

(i) $\mathrm{z}=0.8 \mathrm{~m}$

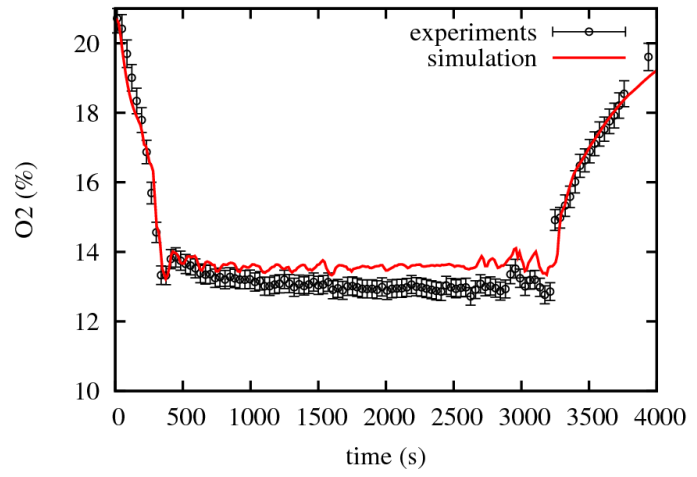

(ii) $\mathrm{z}=3.3 \mathrm{~m}$

Figure 10: Evolution in time of the oxygen concentration in two points (lower and upper part)

\section{CONCLUSION}

ISIS implements a coherent, proven set of models capable of simulating fire development in open environments, as well as in large confined and ventilated enclosures, similar to those in a nuclear facility. Good agreement is obtained for the Prisme Source test for the ventilation, the gas and wall temperatures and the oxygen concentration.

The work on ISIS progresses along two directions to improve the code: physical modelling and numerical schemes. For the first part, many works are currently developed: 
- the evaluation of the mass loss rate in confined domain in order to have a predictive code. First works give encouraging results (Nasr, 2010) where the mass loss rate is computed from the sum of fluxes.

- the production and the oxidation of soot play an important role on the combustion and radiative transfer. It is important to develop accurate models which are available in confined domain.

- the turbulence modelling: an approach by Large Eddy Simulation is in progress in ISIS.

The second part is oriented to have efficient numerical schemes for structured and unstructured meshes. The aim is to have a code able to compute fire for instance in compartment with a complex geometry as there is in nuclear facilities.

\section{REFERENCES}

American Institute of Aeronautics and Astronautics. Guide for the Verification and Validation of Computational Fluid Dynamics Simulations. AIAA G-077-1998 (1998).

G. Ansanay-Alex, F. Babik, J. C. Latché and D. Vola, "An L2-stable approximation of the NavierStokes convection operator for low-order non-conforming finite elements", International Journal for Numerical Methods in Fluids, published online (2010).

L. Audouin, L. Chandra, J-L Consalvi, L. Gay, E. Gorza, V. Hohm, S. Hostikka, T. Ito, W. KleinHessling, C. Lallemand, T. Magnusson, N. Noterman, J.S. Park, J. Peco, L. Rigollet, S. Suard and P. Van-Heeso, "Quantifying differences between computational results and measurements in the case of a large-scale well-confined fire scenario", submitted in Nuclear Engineering and Design (2010).

F. Babik, T. Gallouët, J.-C. Latché, S. Suard and D. Vola, "On two fractional step finite volume and finite element schemes for reactive low Mach number flows", The International Symposium on Finite Volumes for Complex Applications IV - Problems and Perspectives, Marrakech (2005).

J. C. Chai, H. S. Lee, and S. V. Patankar, "Finite volume method for radiation heat transfer", Journal of Thermophysics and Heat Transfer, 8(3):419-425 (1994).

G. Cox, Combustion fundamentals of fire, Academic press (1995).

A. Majda and J. Sethian, "The derivation and numerical solution of the equations for zero Mach number combustion”, Combustion Science and Technology, 42:185-205 (1985).

A. Nasr, S. Suard, J.-P. Garo, H. El-Rabii and L. Gay, "Influence of environmental conditions on the mass loss rate in confined and mechanically-ventilated compartment fires", 12th international conference on fire science and engineering, Nottingham (2010)

W.L. Oberkampf, T.G. Trucano and C. Hirsch, "Verification, Validation, and Predictive Capability in Computational Engineering and Physics", Verification and Validation for Modeling and Simulation in Computational Science and Engineering Applications (2002).

Physical Modelling in ISIS, https://gforge.irsn.fr/gf/project/isis/docman (2010)

T. Poinsot and D. Veynante, Theoretical and numerical combustion, Edwards (2001).

Hugues Pretrel, Philippe Querre and Marc Forestier, "Experimental study of burning rate behaviour in confined and ventilated fire compartment", 8th International Symposium on Fire Safety Science, Pekin (2004). 
G. D. Raithby and E. H. Chui, "A finite-volume method for predicting a radiant heat transfer in enclosures with participating media", Journal of Heat Transfer (Transactions of the ASME), 112:415423 (1990).

P.J. Roache, Verification and Validation in Computational Science and Engineering. Hermosa Publisher (1998).

T. F. Smith, Z. F. Shen and J. N. Friedman, "Evaluation of coefficients for the weighted sum of gray gases model", Journal of Heat Transfer (Transactions of the ASME), 104:602-608 (1982).

S. Suard, L. Audouin, F. Babik, L. Rigollet and J.C. Latché, "Verification and Validation of the ISIS CFD Code for Fire Simulation", ISO/TC 92/SC4 Workshop on Assessment of Calculation Methods in FSE, San antonio, TX, USA (2006).

Validation of ISIS, https://gforge.irsn.fr/gf/project/isis/docman (2010) 